\title{
Head movements based control of an intelligent wheelchair in an indoor environment
}

\author{
Ericka Janet Rechy-Ramirez, Huosheng Hu and Klaus McDonald-Maier \\ School of Computer Science \& Electronic Engineering, University of Essex, Colchester CO4 3SQ, U.K. \\ Email: ejrech@essex.ac.uk, hhu@essex.ac.uk,kdm@essex.ac.uk
}

\begin{abstract}
This paper presents a user-friendly human machine interface (HMI) for hands-free control of an electric powered wheelchair (EPW). Its two operation modes are based on head movements: Mode 1 uses only one head movement to give the commands, and Mode 2 employs four head movements. An EEG device, namely Emotiv EPOC, has been deployed in this HMI to obtain the head movement information of users. The proposed HMI is compared with the joystick control of an EPW in an indoor environment. The experimental results show that Control Mode 2 can be implemented at a fast speed reliably, achieving a mean time of 67.90 seconds for the two subjects. However, Control Mode 1 has inferior performance, achieving a mean time of $\mathbf{1 5 3 . 2 0}$ seconds for the two subjects although it needs only one head movement. It is clear that the proposed HMI can be effectively used to replace the traditional joystick control for disabled and elderly people.
\end{abstract}

Keywords: Emotiv, human machine interface, electric powered wheelchair, hands-free control, head movement detection.

\section{INTRODUCTION}

The current electric powered wheelchairs (EPWs) are mostly joystick-driven, and cannot fully meet the need of the disabled and elderly people whose autonomies are seriously affected by decline in their motor function and cognitive performance. Up to now, various hands-free HMIs have been developed for the disabled and elderly people to control EPWs by using shoulder, head and tongue motion, as well as eye tracking. Jia et al. [7] developed a visual based HMI for controlling a wheelchair by head gestures which were recognized by detecting the position of the nose on user's face. Gajwani and Chhabria [2] used eye tracking and eye blinking obtained by a camera mounted on a cap to control a wheelchair. However, the performances of these HMIs are likely affected by environmental noises such as illumination, brightness, and the camera position. Additionally, eye tracking may force and affect the vision of the user, causing tiredness and dizziness.

On the other hand, some researchers have used electromyography signal (EMG) for controlling wheelchairs by performing certain shoulder movements. Han et al. [4] used four EMG electrodes on the Stenocleidomastoid muscle in order to detect three shoulder movements (both shoulders up, right shoulder up and left shoulder up), achieving an average success rate of $91.2 \%$. While Moon et al. [8] only used two EMG electrodes to detect shoulder movements in order to control the wheelchair. Unfortunately some disabled people may not be able to move their shoulders and bodies, alternative ways are required. Huo and Ghovanloo [6] operated a wheelchair by using tongue movements, in which the movement data was obtained from a magnetic tracer on the tongue. This is a little invasive for long-term usage since the user should receive a tongue piercing embedded with the magnetic tracer. Palankar et al. [9] controlled a wheelchair by using a mounted robotic arm in a simulated environment. The robotic arm was operated by means of a P300 brain computer interface (BCI), in which the user was able to control the motion of the arm and chair by focusing attention on a specific character on the screen. Nevertheless, the response time of the BCI needs to be improved for a real world.

Recently, a new EEG sensor, Emotiv EPOC, has been available on the market to provide potential applications on hands-free HMIs. It has three suites: 'cognitiv suite' to detect thoughts, 'expressiv suite' to detect facial expressions and 'affectiv suite' to detect emotions, as well as a gyroscope to detect head movements. In [3], it was used to recognize four trained muscular events to steer a tractor: (i) eyes looking to the right and jaw opened, (ii) eyes looking to the right and jaw closed, (iii) eyes looking to the left and jaw opened, and (iv) eyes looking to the left and jaw closed. Carrino et al. [1] developed a system, namely "Virtual Move", which allows users to navigate through Google Street View (GSV) using head movements, facial expressions, thoughts and emotional states.

This paper proposes a novel HMI for hands-free control of an electric powered wheelchair based on an EEG sensor called Emotiv EPOC, which can detect head movements. It has two modes: one uses only one head movement to control the wheelchair and the other one uses four head movements. Both control modes obtain the motion data from the gyroscope of the Emotiv EPOC headset. Two healthy subjects have operated a wheelchair using both modes in an indoor environment. It is important to say that once the proposed HMI is running, the user does not need to keep the pose at a specific position. Therefore, the fatigue of the user can be greatly reduced. Both modes provide four control commands: 'going forward', 'turning right', 'turning left' and 'stopping'.

The rest of the paper is organized as follows. Section II presents the research methodology, including a brief description of the Emotiv EPOC headset and the two control modes, namely Mode 1 and Mode 2, used in our HMI. Experimental results and analysis are given in Section III to 
show the feasibility and performance of the two control modes of the proposed HMI in the real-world setting. Finally, a brief conclusion and potential future work are given in Section IV.

\section{Research Methodology}

The proposed two control modes use the gyroscope of the Emotiv EPOC headset in order to detect head movements. As shown in Fig. 1, the Emotiv EPOC headset is a device that measures EEG activity from 14 saline electrodes (plus CMS/DRL references, P3/P4 locations) [5]. These electrodes are arranged according to the 10/20 system, and their locations are AF3, F7, F3, FC5, T7, P7, O1, O2, P8, T8, FC6, F4, F8 and AF4. Besides the gyroscope, the Emotiv Software Development Kit for research includes an API to develop applications using the three different emotiv suites: 'cognitiv', 'expressiv' and 'affectiv' suites. The 'cognitiv suite' recognizes conscious thoughts of the user, the 'expressiv suite' recognizes facial gestures and the 'affectiv suite' recognizes emotional states of the user.
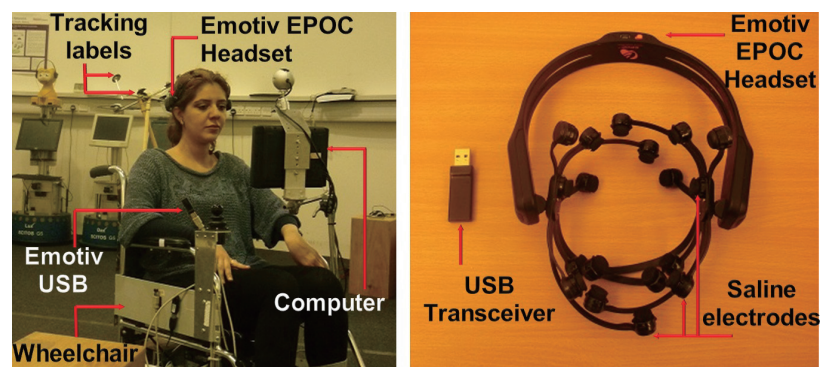

Fig. 1. Intelligent wheelchair setup for subject (Left) and the Emotiv EPOC Headset (Right).

This research is focused on the use of the gyroscope of the Emotiv. The gyroscope has two axes, $\mathrm{X}$ and $\mathrm{Y}$, through which the EmoEngine of the Emotiv sensor gives the position data of the user's head. As shown in the Fig. 2, the $\mathrm{X}$ axis reports horizontal movements of the head, a negative value indicates a left movement of the head and a positive value represents a right movement of the head. On the other hand, the $\mathrm{Y}$ axis identifies vertical movements of the head, a positive value corresponds to an up movement of the head and a negative value indicates a down movement of the head.

\section{A. Four head movements based control mode}

In our proposed HMI, the four head movements based control mode employs the motion data obtained by a twoaxis gyroscope inside the Emotiv sensor to recognize head movements without the need of a camera. In this way, lighting illumination effect is eliminated. As can be seen in the Fig. 3, the user performs a specific head movement, e.g. Up, Down, Right and Left, to issue a control command ('going forward', 'stopping', 'turning right' and 'turning left').

It should be noticed that after giving a control command, the user unconsciously performs the opposite movement of the head in order to return to the original position, which may trigger by mistake the opposite control command. For this
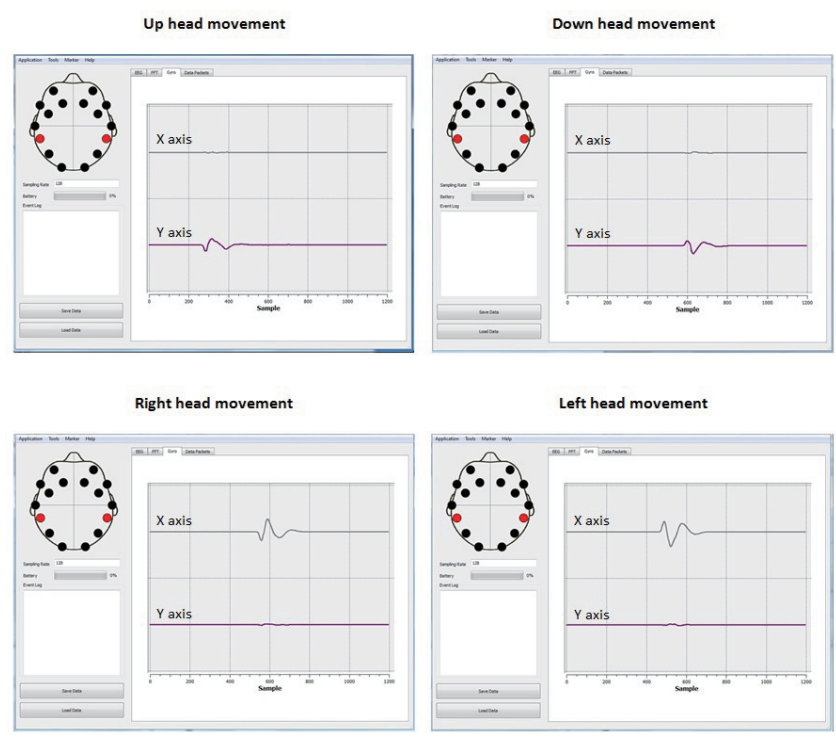

Fig. 2. Gyroscope oscillations according to the head movements.

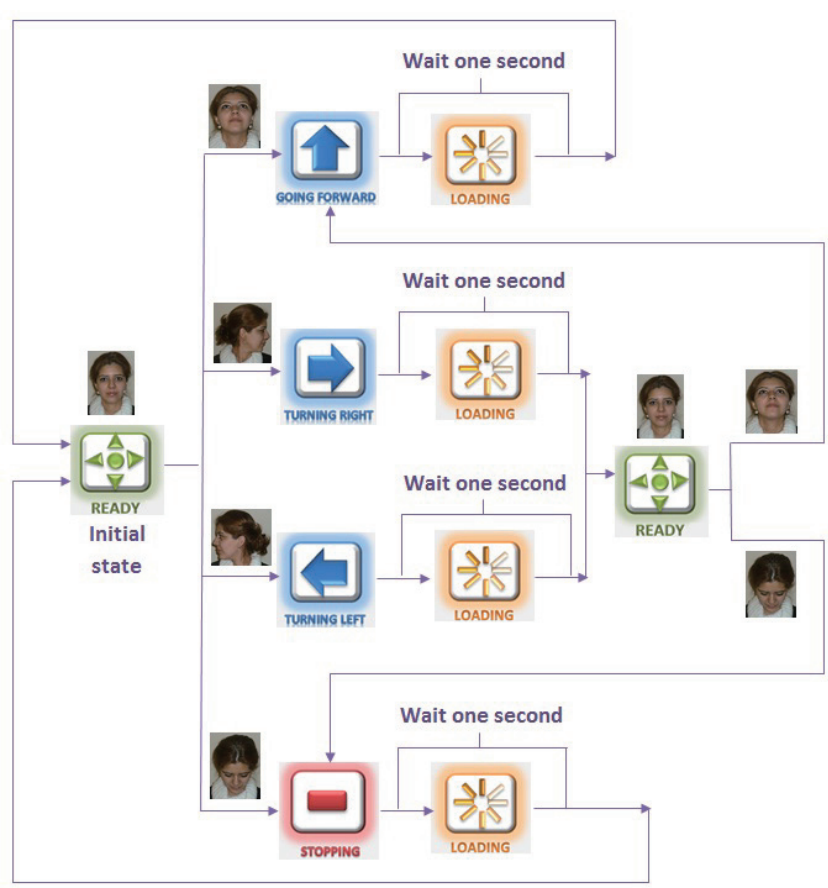

Fig. 3. Algorithm of the four head movements based control mode.

reason, a one-second delay was implemented for the user to return his/her head to the neutral position. Moreover, the $\mathrm{X}$ axis of the gyroscope is changing constantly when the user is turning due to the movement of the wheelchair, therefore the user cannot issue the 'turning right' command when 'turning left' has not completed and vice versa.

The turning angles of the head are determined by constants; nevertheless, as shown in Fig. 4, the graphical user interface of this control mode provides the facility of changing the thresholds for left, right, up and down head movements at execution time, as well as the wheelchair speed. In addition, 


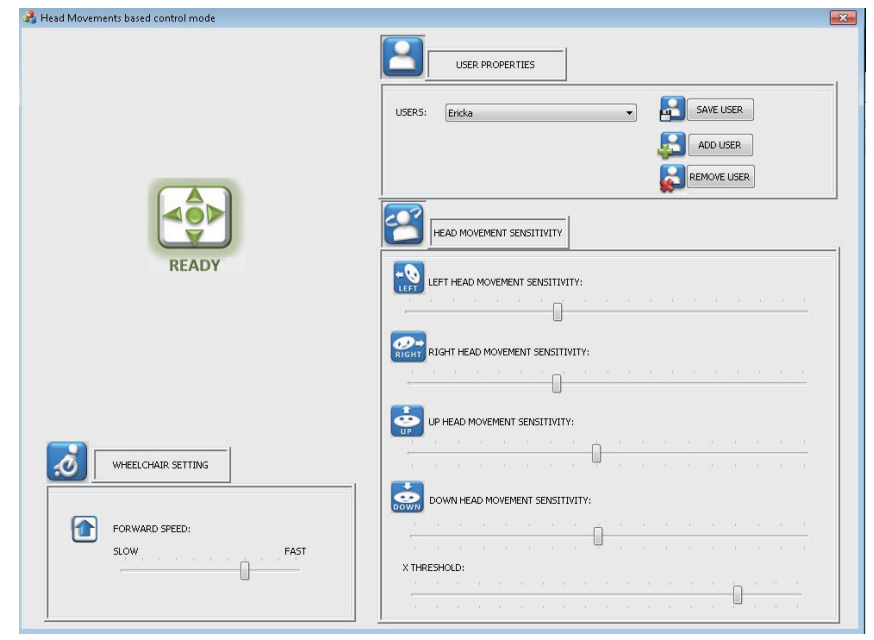

Fig. 4. Graphical user interface of the four head movements based control mode.

this mode offers the options of adding new users, removing users and saving the preferences of the user.

\section{B. One head movement based control mode}

The previous control mode offers four control commands, each command is issued by a different head movement, therefore four head movements are needed for controlling the wheelchair. Nevertheless, some patients may only be able to perform one head movement, for this reason this mode was designed for controlling the wheelchair by using just one head movement. This HMI only employs the 'Y axis' of the gyroscope of Emotiv for the user to achieve 'up' or 'down' head movements. Since only one head movement is used for triggering the four control commands ('going forward', 'turning to the right', 'turning to the left' and 'stopping'), an automation process for displaying the commands is provided in this mode. As can be seen in the Fig. 5, this automation process is based on a rotation technique in which each control command is displayed one second, so the user is able to control the wheelchair by employing just one head movement instead of four.

To use this HMI, firstly the user has to select the desired head movement to control the wheelchair, either 'up head movement' or 'down head movement'. Once that this head movement has been selected, it cannot be changed during the execution of the program and only this movement will be used to control the wheelchair. As can be seen in the Fig. 6, the HMI starts in the state of 'stopping' and below it, a 'loading symbol' is displayed.

In order to display the control commands the user has to perform the head movement selected previously in the HMI. Once this has done, each control command will be displayed for a second. This action was taken into account due to the fact that if the control command symbols are displayed just after the command was triggered, maybe by the time when the desired control command symbol is displayed, the user does not longer need it. So, if the user wants to give a certain control

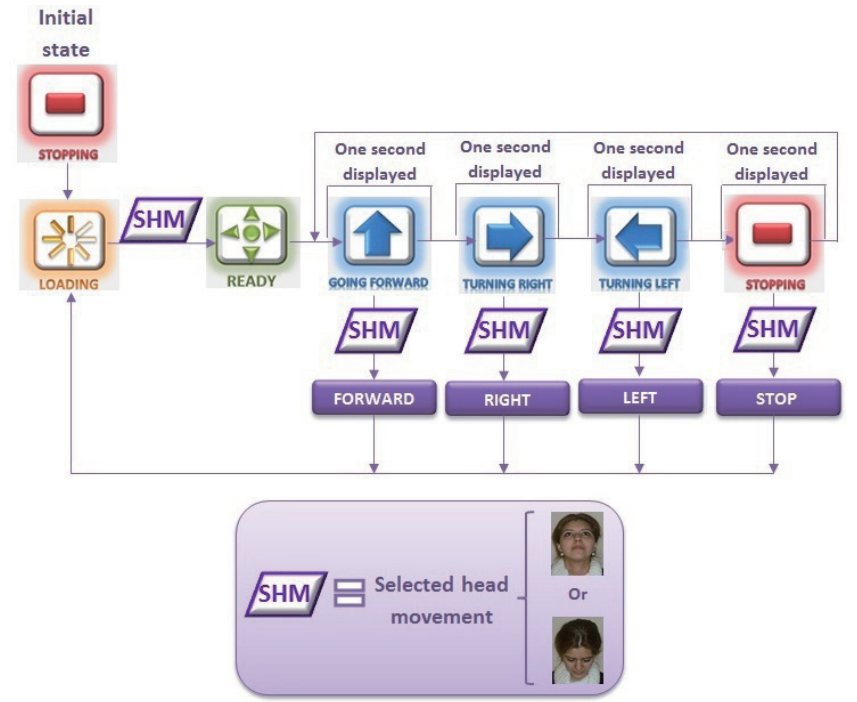

Fig. 5. Algorithm of the one head movement based control mode.

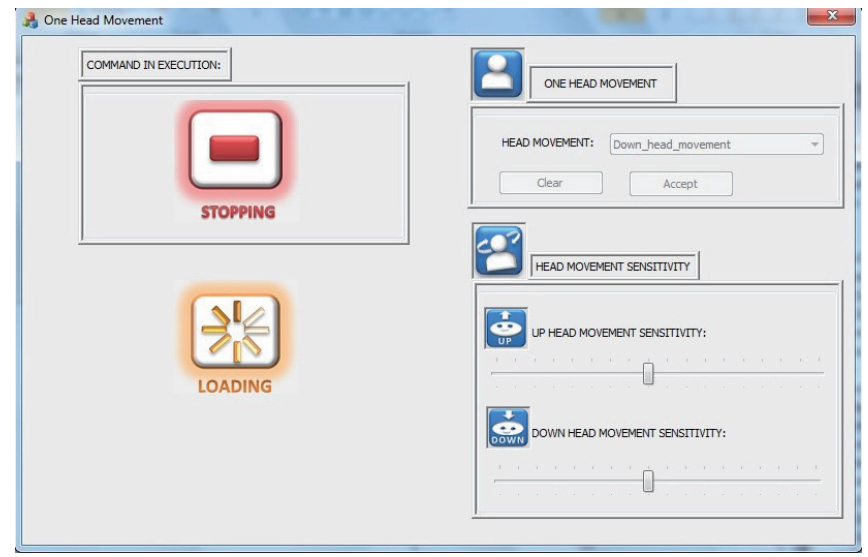

Fig. 6. Graphical user interface of the one head movement based control mode.

command, firstly he needs to perform the head movement to give the instruction of displaying the control commands and then he has to wait for the desired control command until it appears and performs the head movement in order to execute it.

It is important to say that the electrodes of the Emotiv headset are not deployed, as well as the user does not have to maintain the head pose once the control command is issued, so that the user fatigue in both control modes can be minimized.

\section{EXPERIMENTAL RESULTS AND ANALYSIS}

Both control modes of the proposed HMI, 'one head movement'(Control Mode 1) and 'four head movements' (Control Mode 2), and the 'joystick' mode operated with the hand have been tested by controlling a wheelchair in an indoor environment. Two healthy subjects (a female and a male) have operated the wheelchair using the three different control modes. Five experiments were carried out by each control mode. In each experiment, the subject has to follow the route 


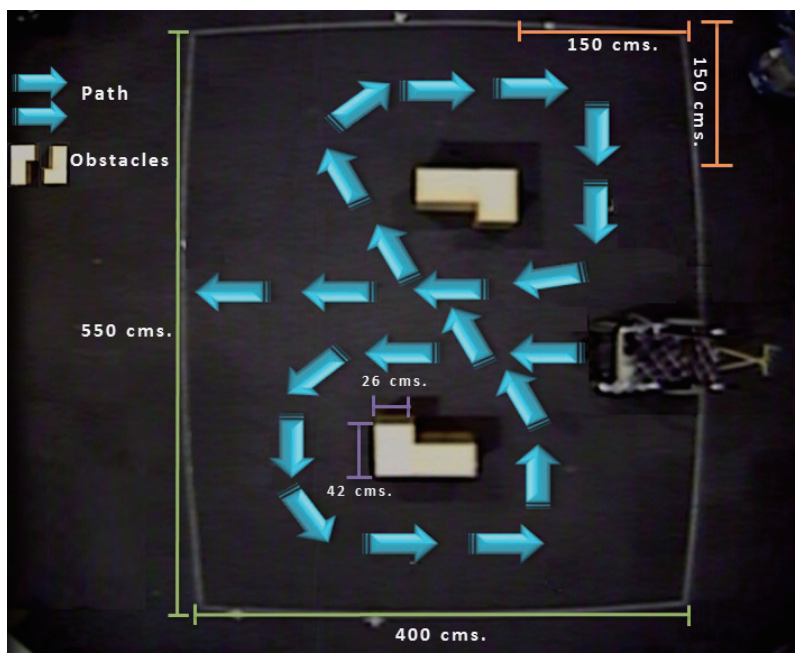

Fig. 7. The route to follow in the indoor environment.

shown in Fig. 7 without hitting the obstacles.

Control Mode 1 was tested using 'slow' and 'medium' speeds, namely 'Mode1-SL' and 'Mode1-MD' for convenience, respectively. Since the response of Control Mode 2 is fast, a 'fast' speed (Mode2-FS) were also deployed in experiments apart from 'slow' (Mode2-SL) and 'medium' (Mode2-MD) speeds. The slow and medium speeds of the wheelchair correspond approximately to a motion of 14.28 and 22.22 centimeters per second, respectively. While the fast speed is approximately equivalent to 27.27 centimeters per second. Finally, the speed employed by the joystick mode was approximately 60 centimeters per second. All speeds were calculated only for the 'going forward' control command.

Traveling times and trajectories were recorded. Means and standard deviations of the traveling times were calculated. The position of the wheelchair was obtained by a Vicon system that tracks the five markers attached to the wheelchair. To achieve a good performance of both control modes, the Emotiv sensor has to be attached properly to the user's head, for this reason a head band was used. In both control modes, the electrodes were not deployed.

Fig. 8 shows the times used by Subject 'A' to travel the route five times per control mode. Subject 'A' had experience on using all control modes at the moment of doing the experiments. As can be seen, the fastest control mode was the joystick, it took less than 30 seconds to finish the path. The second fastest control mode was the 'Mode2-FS', which took 71 seconds at the worst time and 64 seconds at the best time. The 3rd fastest control mode was 'Mode2-MD', finishing the path in all the experiments between 84 seconds and 96 seconds. On the other hand, although 'Mode2-SL' is slightly faster than 'Mode1-MD', they had almost similar times (141 and 136 seconds) in two experiments (1 and 5). The biggest difference between them was only about 24 seconds in the rest of the experiments. Finally, it took more than 170 seconds to finish the route in all the experiments at 'Mode1-SL'.

Fig. 9 shows the times achieved by Subject 'B' repeating the

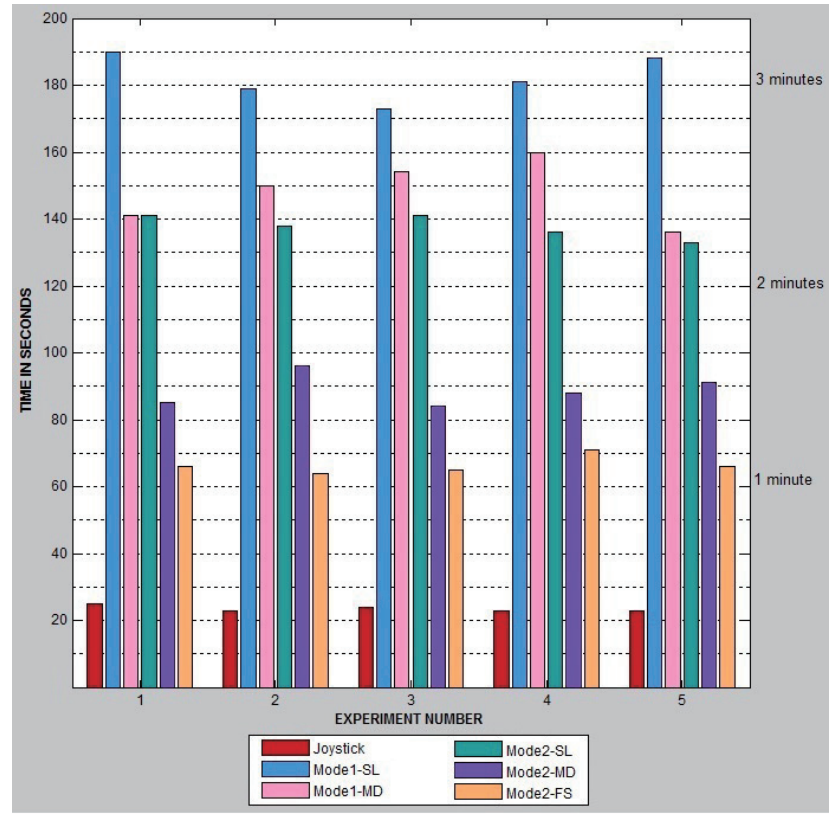

Fig. 8. Time in seconds for Subject 'A' following the route for five times using three different control modes.

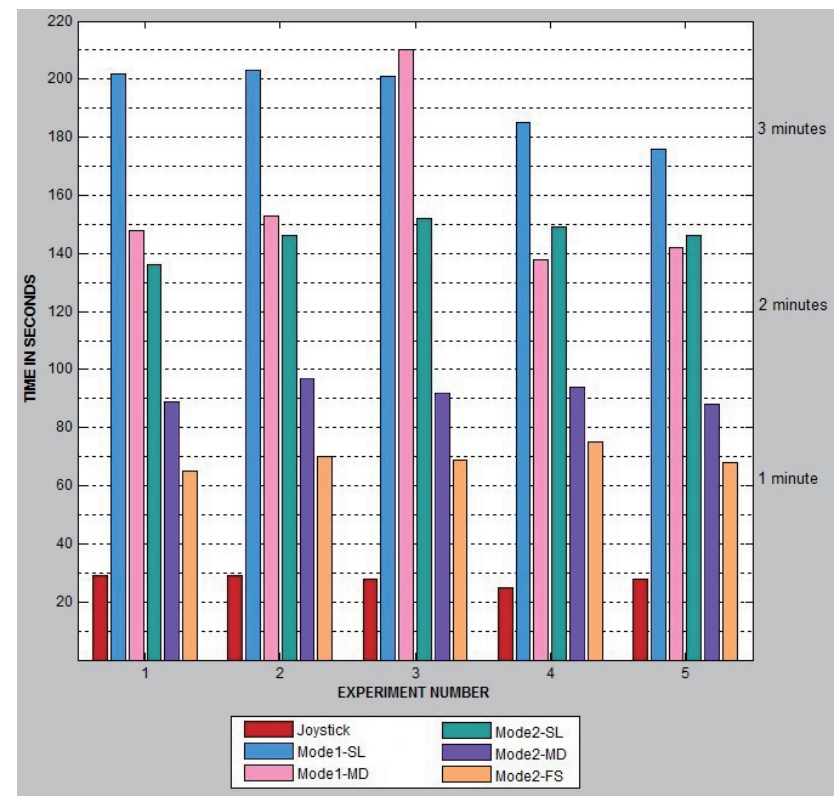

Fig. 9. Time in seconds for Subject 'B' following the route for five times using three different control modes.

route five times per each control mode. Before doing experiments, Subject 'B' did the route once with all control modes. As in the case of Subject 'A', the 'joystick', the 'Mode2-FS' and the 'Mode2-MD' were the three fastest control modes, being 29, 75 and 97 seconds for their worst times, and 25, 65 and 88 seconds for their best times, respectively. In spite of the fact that 'Mode1-MD' is supposed to be faster than 'Mode2SL', which only happened in two experiments of five. Even though, in the experiment number three, the slowest control 


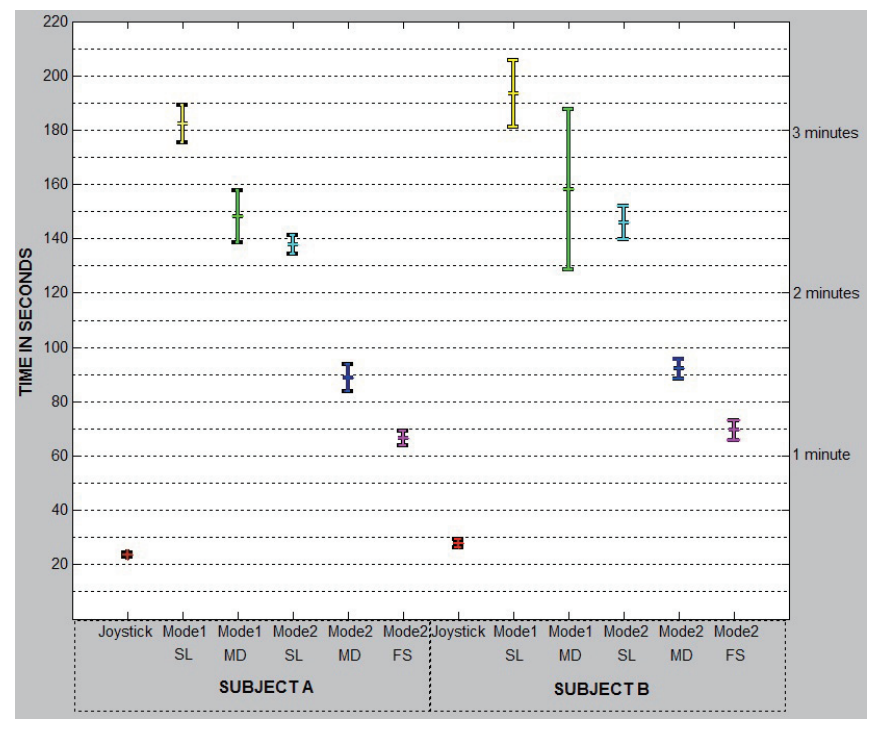

Fig. 10. Means and standard deviations of the traveling times for Subjects 'A' and 'B' using three control modes.

mode for Subject 'B' was 'Mode1-MD', finishing the route in 210 seconds against 201 seconds for 'Mode1-SL'. In four experiments, the slowest control mode for Subject 'B' was 'Mode1-SL', being its worst time 203 seconds and its best time 176 seconds.

Fig. 10 shows the means and standard deviations of the traveling times for Subjects 'A' and 'B' at each control mode. It can be seen that 'joystick', 'Mode2-FS' and 'Mode2-MD' have similar means and standard deviations of the traveling times between two users. The traveling times of both subjects using these three control modes are clustered closely around their means 23.60 and 27.80 for 'joystick', 66.40 and 69.40 for 'Mode2-FS', and 88.80 and 92 for 'Mode2-MD' with standard deviations of 0.89 and 1.64 for 'joystick', 2.70 and 3.64 for 'Mode2-FS', and 4.86 and 3.67 for 'Mode2-MD'. Note that 'Mode1-SL' has the largest means of traveling times for both users (182.20 and 193.40 seconds), and 'Mode1-MD' has the largest standard deviations between two users, i.e. 9.70 and 29.51 respectively.

Fig. 11 shows the boxplots of the traveling times of both subjects in the three different control modes. Although the medians of the traveling times for Subject 'A' are smaller than the medians of the traveling times for Subject 'B' among three control modes, their minimum traveling times are almost the same. The maximum traveling times of three control modes are almost similar between both subjects. However, the maximum traveling times for Subject 'B' in three control modes are greater than the maximum traveling times for Subject ' $A$ ' corresponding to these control modes.

As can be seen in the Figs. 12 and 13, both subjects were capable of following the route shown in Fig. 7, without hitting the obstacles using the three control modes at different speeds. Nevertheless, the survey that was answered by both subjects indicates that the favorite control mode after the joystick

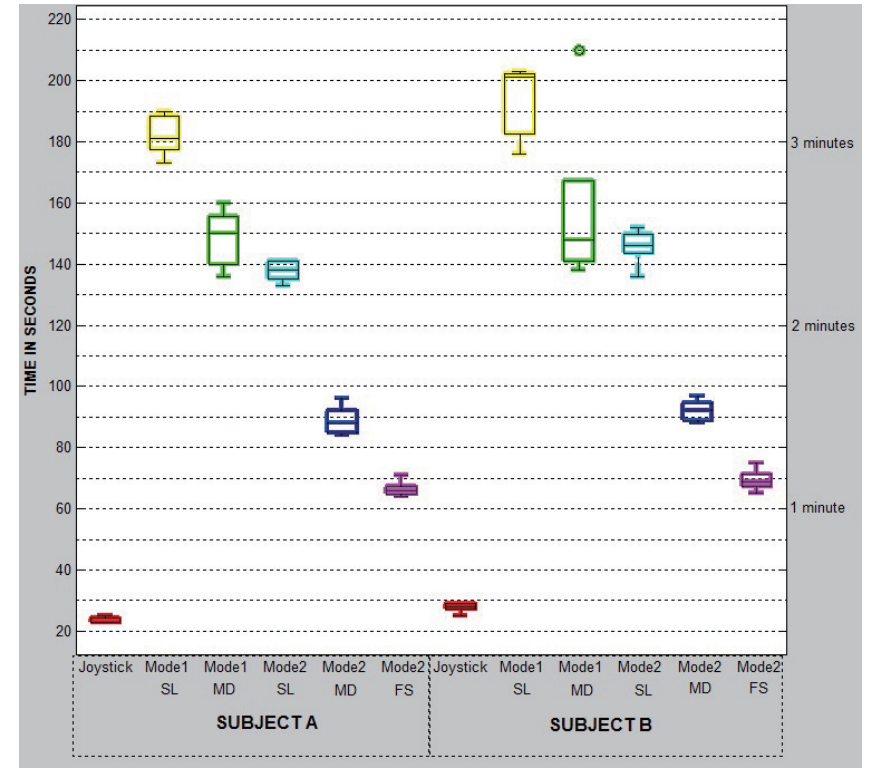

Fig. 11. Traveling times of Subjects 'A' and 'B' at three different control modes

control mode was 'Mode2-FS'. With respect of Control Mode 1, both users prefer 'Mode1-SL' over 'Mode1-MD' due to the fact that they feel more confident at the slow speed to give the control commands. For all these reasons, it is clear that the 'four head movements based control mode' with a fast speed is reliable for controlling a wheelchair, and the 'one head movement based control mode' should be run at a slow speed.

\section{CONCLUSIONS AND FUTURE WORK}

In this paper, a novel head gestures based HMI is proposed for hands-free control of EPWs. It uses head movements detected by the motion data obtained from the gyroscope of an Emotiv sensor. It has two control modes, one mode uses only one head movement ('up' or 'down') and the other one employs four head movements ('up', 'down', 'right' and 'left'). Four control commands were implemented, namely 'going forward', 'turning right', 'turning left' and 'stopping'. In both control modes, the user does not have to maintain the head movement during the control command. Experimental results show that the proposed HMI is reliable for controlling a wheelchair.

The future work will be focused on the implementation of a facial expressions based mode using the Emotiv sensor for controlling a wheelchair, in which the user can choose his/her most comfortable facial expressions to generate the control commands.

\section{Acknowledgements:}

The authors gratefully acknowledge the support of the UK EPSRC Global Engagements grant EP/K004638/1 and the EU Interreg IV A 2 Mers Seas Zeeën Cross-border Cooperation Programme SYSIASS project: Autonomous and Intelligent Healthcare System (http://www.sysiass.eu/). The 1st author has been supported by the Conacyt studentship of the Mexican 


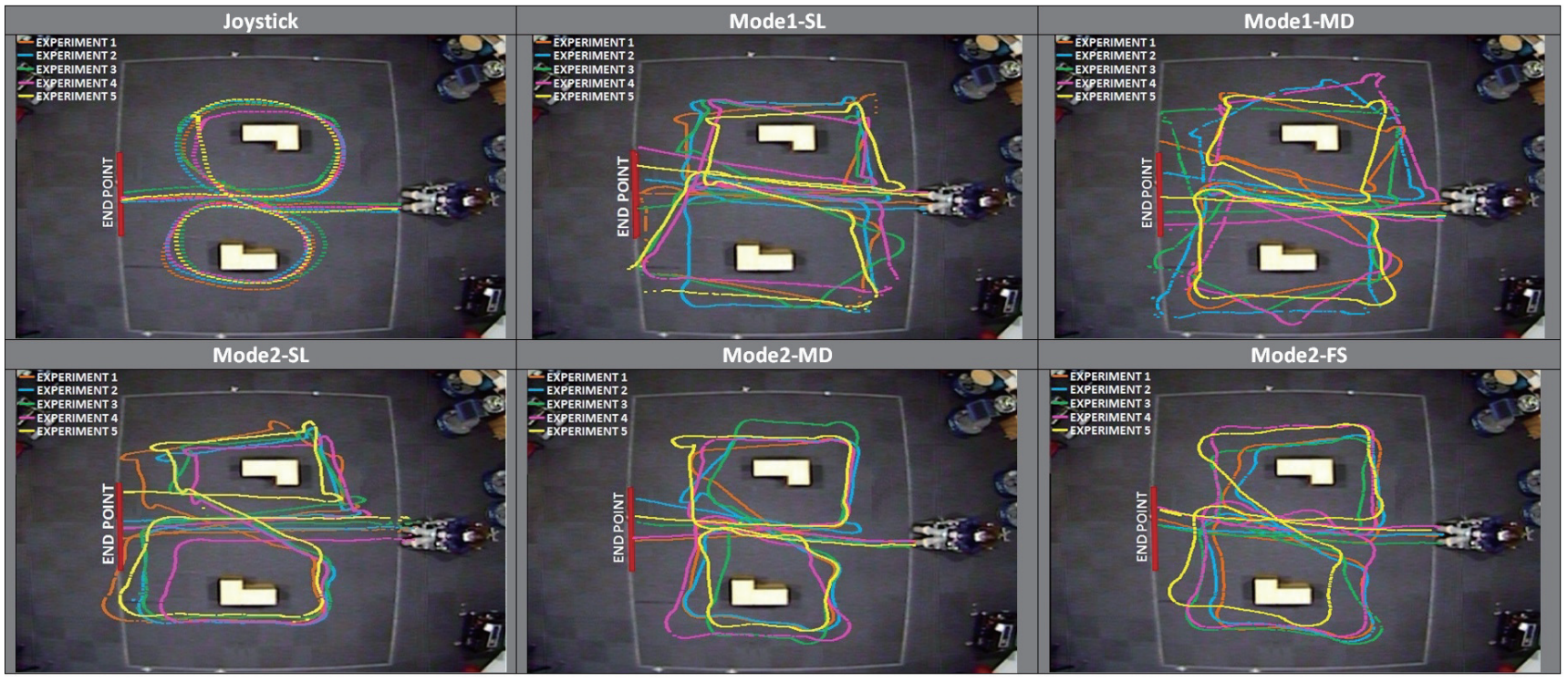

Fig. 12. Trajectories of Subject 'A' employing the three control modes.

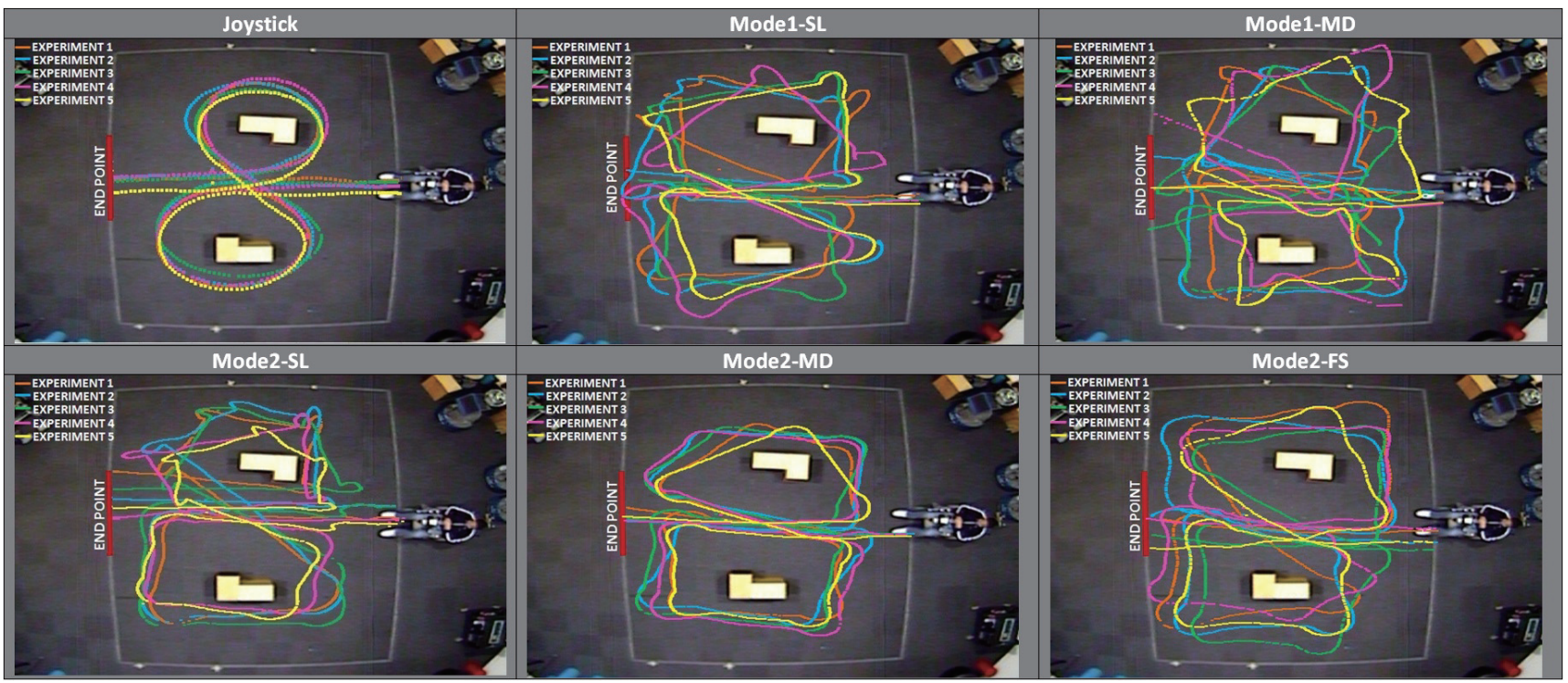

Fig. 13. Trajectories of Subject 'B' employing the three control modes.

Government. Our thanks also go to Robin Dowling for his technical support during the research.

\section{REFERENCES}

[1] F. Carrino, J. Tscherrig, E. Mugellini, O. Abou Khaled, and R. Ingold. Head-computer interface: a multimodal approach to navigate through real and virtual worlds. Human-Computer Interaction. Interaction techniques and environments, pages 222-230, 2011.

[2] P.S. Gajwani and S.A. Chhabria. Eye motion tracking for wheelchair control. International Journal of Information Technology, 2(2):185-187, 2010.

[3] J. Gomez-Gil, I. San-Jose-Gonzalez, L.F. Nicolas-Alonso, and S. AlonsoGarcia. Steering a tractor by means of an EMG-based human machine interface. Sensors, 11(7):7110-7126, 2011.

[4] J.S. Han, Z. Zenn Bien, D.J. Kim, H.E. Lee, and J.S. Kim. Humanmachine interface for wheelchair control with EMG and its evaluation. In Proc. of the 25th Annual Int. Conf. of the IEEE Engineering in Medicine \& Biology Society, volume 2, pages 1602-1605. IEEE, 2003.
[5] http://www.emotiv.com/researchers/. Emotiv software development kit (sdk) for research.

[6] X. Huo and M. Ghovanloo. Using unconstrained tongue motion as an alternative control mechanism for wheeled mobility. IEEE Transactions on Biomedical Engineering, 56(6):1719-1726, 2009.

[7] P. Jia, H.H. Hu, T. Lu, and K. Yuan. Head gesture recognition for hands-free control of an intelligent wheelchair. Industrial Robot: An International Journal, 34(1):60-68, 2007.

[8] I. Moon, M. Lee, J. Chu, and M. Mun. Wearable EMG-based HCI for electric-powered wheelchair users with motor disabilities. In Proceedings of the 2005 IEEE International Conference on Robotics and Automation, pages 2649-2654. IEEE, 2005.

[9] M. Palankar, K.J. De Laurentis, R. Alqasemi, E. Veras, R. Dubey, Y. Arbel, and E. Donchin. Control of a 9-DoF wheelchair-mounted robotic arm system using a P300 brain computer interface: Initial experiments. In Robotics and Biomimetics, 2008. ROBIO 2008. IEEE International Conference on, pages 348-353. IEEE, 2008. 